\title{
Norois
}

Environnement, aménagement, société

$245 \mid 2017$

Adapter les territoires aux changements climatiques : transition urbanistique et aménagement de l'espace

\section{Vers des politiques de déplacements urbains plus cohérentes}

Towards more coherent urban transport policies

\section{Frédéric Héran}

\section{OpenEdition}

\section{Journals}

Édition électronique

URL : http://journals.openedition.org/norois/6242

DOI : $10.4000 /$ norois. 6242

ISBN : 78-2-7535-7465-6

ISSN : $1760-8546$

\section{Éditeur}

Presses universitaires de Rennes

\section{Édition imprimée}

Date de publication : 31 décembre 2017

Pagination : 89-100

ISBN : 978-2-7535-7456-4

ISSN : 0029-182X

\section{Référence électronique}

Frédéric Héran, «Vers des politiques de déplacements urbains plus cohérentes », Norois [En ligne], 245 | 2017, mis en ligne le 31 décembre 2019, consulté le 07 janvier 2021. URL : http:// journals.openedition.org/norois/6242 ; DOI : https://doi.org/10.4000/norois.6242 


Presses
Universitaires
de Rennes
www.pur-editions.fr

\title{
Vers des politiques de déplacements urbains plus cohérentes
}

\author{
Towards more Coherent Urban Transport Policies
}

\author{
Frédéric Héran
}

CLERSE (Centre lillois d'études et de recherches sociologiques et économiques), UMR 8019 du CNRS, Cité scientifique, 59655 VILLENEUVE-D’AscQ cedex. (frederic.heran@univ-lillel.fr)

\begin{abstract}
Résumé : Sommées de réduire le trafic automobile, les politiques de déplacements urbains ont bien du mal à proposer des solutions cohérentes, en cherchant à satisfaire tout le monde. Elles s'efforcent de sortir du «tout automobile », en tombant parfois dans le «tout transports publics» qui n'est pas plus réaliste. Pour se dégager de cette approche sectorielle, l'article propose de développer une approche systémique qui prenne réellement en compte l'ensemble des modes de déplacement, en analysant notamment la manière dont les modes se concurrencent et génèrent des externalités négatives. Issues de cette approche systémique, les politiques de modération de la circulation automobile qui ne cessent de s'étendre depuis 50 ans dans tous les pays développés pourraient constituer le socle de politiques de déplacements plus cohérentes.
\end{abstract}

\begin{abstract}
Required to reduce car traffic, urban transport policies have great difficulties in proposing coherent solutions, seeking to satisfy everyone. They strive to get out of the "all-car policies", sometimes falling into the "all-public transport policies" which is no more realistic. In order to escape from this sectoral approach, the article proposes to develop a systemic approach that takes into account all transport modes, in particular by analyzing how modes compete with each other and generate negative externalities. As a result of this systemic approach, the traffic calming policies, which have continue to grow over the past 50 years in all developed countries, could provide the basis for more coherent transport policies.
\end{abstract}

Mots clés : Changement climatique - renouvellement urbain - ville durable - transport collectif - mobilité

Keywords: Climate change - urban renewal - sustainable city - public transport - mobility

\section{INTRODUCTION}

Depuis 35 ans, le législateur s'est efforcé d'amener progressivement les villes à développer des politiques de déplacement plus cohérentes, tenant compte de tous les modes. En 1982, la loi d'orientation des transports intérieurs (LOTI) proposait aux villes de réaliser des "plans de déplacements urbains » (PDU) pour programmer et coordonner la politique de déplacements et de stationnement.
Puis en 1996, la loi sur l'air et l'utilisation rationnelle de l'énergie (LAURE) rendait les PDU obligatoires pour les villes de plus de 100000 habitants, avec un objectif clairement affiché de "réduction du trafic automobile ». Enfin en 2014, la loi de modernisation de l'action publique territoriale et d'affirmation des métropoles (MAPTAM) transformait les autorités organisatrices des transports urbains (AOTU) en autorités organisatrices de la mobilité (AOM) char- 
gées d'articuler toutes les formes de mobilité dont les « usages partagés des véhicules » et les «modes de déplacement non motorisés ».

Pourtant, force est de constater qu'aujourd'hui, la façon d'aborder les déplacements urbains reste marquée par une approche que l'on peut qualifier de sectorielle. À des politiques donnant la priorité à l'automobile ont succédé souvent dans les grandes villes des politiques plus centrées sur les transports publics, mais toujours monomodales : les autres modes de déplacement - marche, vélo, deux-roues motorisés (2RM), covoiturage, autopartage - n'étant le plus souvent traités que de façon assez marginale, à travers surtout la création de pôles d'échange multimodal. Ces modes représentent cependant, selon les agglomérations et selon le centre et la périphérie, 10 à $30 \%$ des distances parcourues, 20 à $60 \%$ des déplacements effectués et souvent plus de la moitié du temps consacré à la mobilité quotidienne ${ }^{1}$ (Certu, 2012).

Si l'intégration de tous les modes de déplacement progresse, elle reste toutefois assez lente. Dans son bilan des PDU réalisé en 2006, JeanMarc Offner dénonce l'absence de vision : « des référentiels absents, obsolètes ou partiels », "Pas de stratégie affichée, peu de solutions pertinentes, pas de problèmes structurants! »(Offner, 2006). En pratique, beaucoup d'élus développent un discours rassembleur en considérant qu' « il ne faut pas opposer les modes de déplacement », ni «stigmatiser » ou " culpabiliser » les automobilistes en leur « faisant la morale », que « les divers modes sont complémentaires » et qu’il suffit de trouver leur « domaine de pertinence ». Entérinant ce discours, certains chercheurs considèrent ouvertement qu'il n'y a pas d'alternative réelle à l'automobile hors des zones denses des grandes villes (Paul-Dubois-Taine, 2010; Buhler, 2015; Masboungi, 2015; Flonneau et Orfeuil, 2016).

Cet article vise à comprendre d'où vient ce décalage entre les ambitions affichées, notamment dans

1. Quel critère prendre en compte pour comparer les modes de déplacement? La réponse dépend de l'objectif poursuivi. Pour l'ingénieur qui construit ou gère les infrastructures, la distance parcourue est fondamentale, car la capacité de la voirie et son niveau de congestion en dépendent. Pour l'économiste ou l'urbaniste pour qui un déplacement sert d'abord à effectuer une activité à destination, qu'importe la distance, le nombre de déplacements - et donc les parts modales - est le plus pertinent : c'est le parti retenu ici. Pour le sociologue ou le spécialiste des accidents, le temps passé à se déplacer ou le temps d'exposition au risque est le meilleur critère. les PDU, et la réalité assez différente des politiques de déplacements. Faut-il en prendre acte, comme le proposent les auteurs précités, renoncer à toute ambition et réhabiliter, voire encourager, l'usage de la voiture partout où elle semble le mode le plus pertinent? Ou bien s'agit-il seulement de difficultés passagères? Notre hypothèse est que c'est d'abord une question de méthode. Nous manquons de recul historique pour comprendre la situation et les enjeux d'aujourd'hui. Nous ne prenons pas non plus la mesure de ce qu'exige une approche systémique et non plus sectorielle des déplacements urbains. Nous ne voulons pas voir la concurrence que se livrent les modes de déplacement, ni combien certains modes génèrent beaucoup plus d'externalités négatives que d'autres.

L'article reprend d'abord l'approche sectorielle des déplacements urbains en rappelant sa traduction dans les faits et ses conséquences, puis lui oppose une approche systémique nettement plus ambitieuse mais non sans limites, pour montrer enfin que cette approche systémique impose un changement de paradigme. Il insistera sur la dimension spatiale des questions évoquées.

\section{L'APPROCHE SECTORIELLE DES DÉPLACEMENTS URBAINS}

Pour comprendre pourquoi les déplacements urbains continuent aujourd'hui d'être abordés sans réelle politique d'ensemble, il convient de revenir sur l'origine des solutions et des outils mis en place il y a plus de 50 ans dans une logique typiquement sectorielle.

\section{L'avènement du « tout automobile »}

Dans la France d'après-guerre, l'usage des 2RM puis de l'automobile se démocratise à un rythme accéléré, porté par une population enthousiaste à l'idée d'accéder à plus de liberté dans ses déplacements, en échappant tout à la fois aux contraintes des transports publics et à la fatigue de la marche et du pédalage. Dans les agglomérations, l'urgence des élus comme des techniciens est de faire place à la voiture. Mais dès les années 1950, les spécialistes du trafic comme les urbanistes s'inquiètent de l'énorme consommation d'espace qu'exige l'automobile en routes et parkings et de l'inévitable augmentation 
de la congestion qui en découle. Il apparaît tout simplement impossible d'adapter complètement la ville à l'automobile, faute de place disponible (Smeed, 1961). Au cours des années 1960, d'autres spécialistes remarquent qu'environ $30 \%$ de la population seront toujours dans l'incapacité d'accéder à l'automobile pour des raisons d'âge, de handicap ou de faibles revenus. En conséquence, ils réclament la relance des transports publics jusqu'alors abandonnés à leur sort (Bigey et Schmider, 1971).

C'est pourquoi, en 1971, l'État propose un compromis résumé en un discours normatif destiné aux grandes villes de province et fondé sur quatre « solutions recommandées » étroitement complémentaires : la réalisation de parkings souterrains ou en élévation en centre-ville; la construction d'un réseau autoroutier « largement dimensionné »; l'implantation de transports collectifs en site propre (TCSP) sur les axes d'urbanisation les plus denses, « en surface dans les zones périphériques » et « en sous-sol au centre »; et l'aménagement d'un secteur piétonnier dans les voies commerciales et historiques les plus fréquentées de l'hypercentre. À cela s'ajoutent des «mesures d'exploitation des réseaux urbains » reposant sur un plan de circulation et une organisation du stationnement, indispensables pour « tirer le meilleur parti des réseaux de voirie existants » (Poulit, 1971, p. 67).

Ce discours relève clairement du « tout automobile ». Cette expression française, sans équivalent en anglais, ne signifie pas qu'il soit possible d'utiliser la voiture pour tous les déplacements; elle peut être définie comme la priorité accordée à la voiture en toutes circonstances. Les autres modes de déplacement ont le droit d'exister ou même de se développer, à condition qu'ils n'entravent pas la circulation et le stationnement automobiles.

Ainsi, dans le compromis proposé, les deux-roues sont totalement absents parce que déjà jugés en voie de disparition, alors qu'ils représentent encore à l'époque 10 à $20 \%$ des déplacements dans les grandes villes et bien plus encore dans les villes moyennes. Les aménagements cyclables qu'on leur concèdera parfois, après la crise de l'énergie de 1974, seront destinés à les retirer de la chaussée pour faire place à la voiture. Les piétons ne sont pleinement considérés que dans des « zones piétonnes » restreintes. Partout ailleurs, les trottoirs sont rognés et les traversées de chaussées réduites au strict nécessaire. Les espaces publics sont considérés comme des délaissés (Devillers, 1994). Les transports publics ne sont envisagés qu'à condition qu'ils soient enterrés dans les zones congestionnées, de façon là encore à libérer de la place pour la voiture en surface. Quant aux plans de circulation, ils augmentent d'environ $30 \%$ la vitesse et le volume du trafic, au détriment des modes les plus vulnérables (Gerondeau, 1977).

Les modèles de trafic contribuent aussi à renforcer le tout automobile, parce qu'ils sont incapables de prendre en compte l'impact en retour des infrastructures sur l'urbanisme via l'étalement urbain (Dupuy, 1975). Les usagers profitent, en effet, de déplacements plus rapides non pas pour gagner du temps mais pour aller plus loin (Zahavi, 1973). Des modèles transports-urbanisme sont certes en cours de développement, mais ils réclament de telles quantités de données, difficiles et coûteuses à obtenir ou délicates à rendre cohérentes, qu'ils s'avèrent peu opérationnels (De Lapparent, 2013).

Le tout automobile n'a pas seulement généré un fort étalement urbain (Wiel, 1999), il a aussi contribué au dépérissement des espaces publics étouffés par l'omniprésence de la circulation et plus encore du stationnement, ainsi qu'à la dislocation de la ville par de multiples effets de coupure liés à la fois aux infrastructures routières et aux parkings. Il faut réaliser, en effet, qu'un déplacement en voiture et le stationnement qu'il exige à destination consomme de 50 à 300 fois plus d'espace, selon les hypothèses retenues, qu'un déplacement effectué avec tout autre mode (Héran, 2013).

\section{Les transports publics comme alternative?}

Pour les promoteurs du transport public, l'alternative au tout automobile est ce que l'on pourrait appeler de manière similaire : le « tout transports publics ». L'idée n'est pas de prétendre effectuer tous les déplacements en transports publics, mais de considérer qu'il faudrait leur accorder la priorité en toutes circonstances, qu'il s'agisse de construire les lignes en viaduc, en souterrain ou en site propre avec priorité aux carrefours à feux, de rendre les transports publics gratuits en disqualifiant au passage le vélo ou de leur consacrer des budgets conséquents. L'amélioration de leur vitesse commerciale devient 
la pierre angulaire de cette politique. Les autres modes de déplacement sont considérés comme de simples compléments qu'il convient d'associer en élaborant des politiques d'intermodalité : construction de parcs relais pour les automobilistes et aménagement de pôles d'échanges, création de stations de covoiturage (Paul-Dubois-Taine, 2010 et 2012).

La transformation récente des AOTU en AOM est une illustration de ce basculement vers les transports publics. Il a paru naturel au législateur de confier l'organisation de toutes les mobilités à des autorités habituées jusqu'ici à monter de lourds dossiers de financement de transports publics, puis à suivre les performances des réseaux. Elles manquent pourtant des compétences nécessaires pour traiter les mobilités actives et les usages partagés des véhicules et tendent à ne voir dans ces modes que des solutions mineures et non prioritaires.

De plus, tout comme l'automobile, le transport public contribue à l'étalement urbain. Ce fut le cas avant l'avènement de l'automobile (Wiel, 1999) et cela le redevient aujourd'hui avec l'extension des réseaux de TCSP, même s'il est vrai que cet étalement est bien moins diffus car limité aux alentours des gares et stations. Le Grand Paris Express et « l'urbanisme orienté par le rail » sous-jacent à ce projet pourraient n'avoir guère plus d'efficacité que le maillage de l'Île-de-France par des autoroutes urbaines 50 ans plus tôt (Wiel, 2015).

La plupart des agglomérations ont fini par adopter une "politique duale ", selon une expression utilisée par la FNAUT (Fédération nationale des associations d'usagers des transports), visant à réserver les transports publics aux seules zones denses et à développer l'usage de la voiture en périphérie. De nombreux analystes justifient désormais ce choix. En voici deux exemples récents. Pour Ariella Masboungi, nous vivons aujourd'hui «la montée de l'individu multimodal, la fin de la "guerre des modes" opposant les tenants des transports collectifs à ceux de l'automobile... » (Masboungi, 2015, p. 8). L'ouvrage qu'elle a dirigé distingue « la ville consolidée » où l'urbanisation est « composée autour des espaces publics ", un usage de la voiture strictement encadré et des transports publics performants, à « la ville intermédiaire » où « l'urbanisme moderne a donné une place de choix à la voiture reine » (ibid., p. 11-15). Il s'agit simplement d'entériner cette réalité. Conclusion similaire pour Mathieu Flonneau et Jean-Pierre Orfeuil qui constatent, nombreuses données à l'appui, que la voiture est utilisée massivement hors des zones agglomérées denses, qu'elle pollue de moins en moins (le livre a été écrit avant le scandale Volkswagen et ses développements) et qu'il convient donc d'encourager son usage (Flonneau et Orfeuil, 2016).

Un tel raisonnement correspond à une approche statique de la question des déplacements. Il n'y a pas de mise en perspective historique suffisante, ni de réelle vision à long terme. Certes, les auteurs tiennent compte des solutions techniques émergentes (voiture électrique, voiture autonome, covoiturage, vélo à assistance électrique...), mais ils n'intègrent guère les contraintes environnementales et les nouvelles aspirations sociales. Ainsi, les nuisances des transports sont largement sous-estimées, l'aspiration des populations à être plus actives dans leurs déplacements est ignorée, la remise en cause progressive et mondiale du tout automobile semble méconnue. Il y a confusion entre ce qui est et ce qui devrait être.

\section{Intérêt et limites de l'approche sectorielle}

L'approche sectorielle est issue de la conception cartésienne de la résolution de problèmes. Pour Descartes, il serait plus facile de traiter un problème en isolant ses composantes, puis en les résolvant une par une (cf. le deuxième précepte de son Discours de la méthode, 1637). Cette idée qui semble relever du bon sens s'est peu à peu imposée au cours du XIX ${ }^{e}$ siècle avec le positivisme et reste aujourd'hui très répandue. Des générations de spécialistes ont été formés à cette école et ont développé grâce à elle une expertise considérable dans tous les domaines, en élaborant au passage des outils sophistiqués, d'une grande efficacité. Mais les solutions élaborées dans le cadre d'une approche sectorielle n'ont aucune raison d'aboutir à une cohérence d'ensemble. Apparaissent inévitablement ce que les Anglo-saxons appellent des « inconvénients imprévus » (unexpected drawbacks) et que les francophones préfèrent nommer des « effets pervers ».

Ainsi, les divers spécialistes intervenant dans le domaine des déplacements urbains, de l'aménagement ou de l'urbanisme ont certes acquis de grandes compétences, chacun dans leur domaine, 
mais ont bien du mal à dialoguer. Les ingénieurs qui construisent ou gèrent les infrastructures de transport développent une approche réticulaire, sans se rendre vraiment compte que les réseaux façonnent la ville et contribuent à l'étaler. Les urbanistes et aménageurs qui élaborent les grands projets urbains développent une approche avant tout spatiale et considèrent que les résultats des modèles de trafic sont une simple donnée à prendre en compte.

Les effets pervers qui en découlent ont déjà été bien repérés (Goodwin, 1998 et 2003; Reigner et al., 2013). Nous n'en donnerons qu'un seul exemple. Dans les villes de province, l'investissement dans les TCSP (métros, tramways modernes et bus à haut niveau de service) a été considérable. Pourtant, depuis vingt ans, le ratio dépenses sur recettes n'a cessé de se dégrader et la part modale des transports publics a très peu progressé (Faivre d'Arcier, 2010; Certu, 2012). En examinant la question de plus près, on constate que les solutions visant à améliorer exclusivement l'efficacité des transports publics attirent peu les automobilistes (Certu, 2002; Cerema, 2015). Par exemple, sur la ligne 1 bis du métro de Lille inaugurée en 1990, 7 \% seulement des usagers sont d'anciens automobilistes. Le report modal est bien meilleur quand le TCSP contribue aussi à limiter directement le trafic automobile. Ainsi, dans le cas du Busway, un bus à haut niveau de service inauguré à Nantes en 2006 et qui a permis de requalifier une pénétrante autoroutière de 2,3 km (l'A801) en avenue urbaine à $2 \times 1$ voie, $23 \%$ des usagers utilisaient auparavant une voiture. Une politique de déplacement plus cohérente est toujours plus efficace.

\section{L'APPROCHE SYSTÉMIQUE DES DÉPLACEMENTS URBAINS}

Pour sortir des impasses de l'approche sectorielle, il suffirait donc d'adopter une approche systémique permettant de penser l'articulation entre tous les modes de déplacement, sans ignorer la façon dont ils se concurrencent et les diverses nuisances qu'ils génèrent.

\section{Intérêt et limites de l'approche systémique}

Pour les systémistes, un problème ne peut jamais être totalement circonscrit et encore moins décomposé en ses parties sans risque d'aboutir à des solutions incohérentes, ignorant les liens multiples et évolutifs entre toutes ses composantes et avec l'environnement. Il s'inscrit toujours dans un ensemble d'interrelations qui font système, le système étant lui-même ouvert et en évolution. C'est pourquoi, seule une solution globale ou intégrée, qui tienne compte de cette double dimension systémique et dynamique, a quelques chances de réussir (Le Moigne, 1977). L'approche systémique pose cependant la question des frontières de l'analyse. Où doit s'arrêter la prise en compte des causes et conséquences du problème étudié? On n'est jamais sûr qu'une variable importante n'a pas été oubliée ou sous-estimée à tort. L'approche systémique n'est donc pas une solution miracle. Elle cherche seulement à reculer les limites de l'approche cartésienne.

Dans le domaine des déplacements urbains, l'approche systémique consiste à tenir compte d'emblée de l'ensemble des modes de déplacement et de leurs interrelations. C'est l'ambition de ce que l'on nomme d'habitude l'approche multimodale. Mais ce terme reste assez flou. Quels modes prendre en compte? La plupart du temps, seuls la voiture et les transports publics sont retenus, en considérant - souvent de façon implicite, comme si cela allait de soi - que les autres modes sont négligeables parce qu'ils ne concernent que des déplacements de proximité ou des modes confidentiels.

C'est pourquoi, un nouvel adjectif paraît nécessaire pour caractériser une approche qui prenne réellement en compte tous les modes. Nous proposons de l'appeler « omnimodale» (de omnes signifiant tout en latin). Concrètement, l'approche omnimodale vise à ne pas oublier les piétons, les cyclistes, les 2RM et les usages partagés des véhicules, dont on a rappelé en introduction l'importance en termes de distances parcourues et surtout de nombre de déplacements effectués et de temps passé à se déplacer.

\section{Des modes de déplacement en concurrence}

L'approche omnimodale permet d'aborder la concurrence entre tous les modes. Certes, chaque mode a en principe son « domaine de pertinence ", mais ces domaines tendent à se recouvrir de plus en plus. Les transports publics cherchent à assu- 
rer une desserte de proximité et à irriguer le grand périurbain. Le vélo n'est plus cantonné aux courtes distances avec la création de réseaux structurants et le développement rapide des vélos à assistance électrique (VAE). La voiture tend depuis longtemps à être utilisée pour tous les types de déplacement. La concurrence joue ainsi à de multiples niveaux.

Elle est d'abord inévitable car on sait depuis une cinquantaine d'années que les déplacements se limitent toujours en moyenne à trois ou quatre par jour (Zahavi, 1973). En conséquence, encourager certains modes se fait forcément au détriment des autres. Toute politique de déplacement engendre fatalement des gagnants et des perdants : impossible de satisfaire tout le monde. Certains élus annoncent avec conviction qu'ils veulent augmenter la part modale de tous les modes de déplacement, sans se rendre de l'incohérence de leur propos ${ }^{2}$. Au nom du développement économique et social, de nombreux SCoT - comme celui de Lille réalisé en 2015 - affirment qu'ils veulent tout à la fois réduire la congestion routière en investissant dans le réseau routier, développer les transports publics et encourager l'usage des «modes doux ».

La compétition porte aussi sur l'occupation de l'espace, ressource particulièrement rare en zone dense. L'offre de voirie et de parking est en effet très rigide : il faut de lourds investissements et beaucoup de temps pour l'augmenter, alors que la demande d'espace-temps (mesurée en $\mathrm{m}^{2}$-h) de circulation et de stationnement est extrêmement variable dans le temps et selon les modes, puisqu'elle peut passer, par exemple, de 1 à 300 selon qu'un salarié se rend en bus ou en voiture à son travail. Encourager les modes de déplacement économes en espace évite de disloquer la ville en créant les immenses parkings et les larges voiries que réclame l'automobile (Héran, 2013).

La concurrence concerne tout autant la vitesse et la portée des déplacements. Pour que les automobilistes puissent accéder à la vitesse que leur permet leur véhicule, il faut ségréguer les trafics lents et rapides tant sur les plans horizontal, vertical que temporel : aménager des voies de circulation distinctes, créer des passages dénivelés et autori-

2. Certes, ils n'affirment pas directement qu'ils souhaitent « augmenter la part modale de la voiture », un objectif qui serait incompatible avec le PDU, mais ils tiennent à «faire sauter les bouchons », ce qui revient bel et bien à encourager l'usage de la voiture. ser la traversée successive des différents usagers par des feux de signalisation. Mais la séparation des flux allonge et complique les déplacements et pénalise en premier les usagers non motorisés qui se retrouvent contraints à effectuer des détours, à franchir des dénivelés et à attendre longuement. Ce qui les amène souvent à renoncer à se déplacer ou à se rabattre sur les modes de déplacement motorisés (Héran, 2011a). Les modes se distinguent aussi par leur portée, selon qu'ils sont ou non motorisés. La motorisation tend à disqualifier les modes actifs. Les déplacements lointains ne sont pourtant pas plus utiles que les déplacements de proximité, puisque seule compte l'activité réalisée à destination.

Les rapports de force s'invitent aussi dans le domaine de la sécurité routière. Il existe de très fortes inégalités face au risque d'accident qui va de 1 à 50, selon qu'un usager prend une voiture ou une moto pour circuler en milieu urbain. Car le risque pour soi et le risque pour les autres varient énormément selon l'énergie cinétique des antagonistes. En cas de choc d'un piéton ou d'un cycliste avec un véhicule motorisé, le risque pour soi est démesuré et le risque pour les autres minime, et c'est l'inverse pour l'usager motorisé. Le $2 \mathrm{RM}$ quant à lui est à la fois très vulnérable et dangereux pour les autres.

La lutte est enfin vive pour les financements, non seulement parce que les ressources sont de plus en plus limitées, mais aussi parce que les coûts d'aménagement sont très différents selon les modes de déplacement par personne transportée, en investissement comme en exploitation. Les écarts vont de 1 à 10,30 voire 50 entre des aménagements piétonniers ou cyclables et des aménagements routiers ou destinés aux transports publics.

\section{Des nuisances qui font système}

Les externalités négatives des transports restent globalement peu étudiées et sous-estimées. Certes, des travaux considérables ont été réalisés sur quatre d'entre elles : la pollution atmosphérique, le bruit, la congestion et les accidents. Mais bien d'autres nuisances mériteraient autant d'attention, notamment l'épuisement des ressources non renouvelables, la consommation d'espace par les transports, les effets de coupure des infrastructures en milieu urbain, l'impact des transports sur le paysage, la pollution des eaux et des sols, les îlots de chaleur ou l'artifi- 
cialisation des sols. Cela suppose de parvenir, autant que possible, à les qualifier, à les quantifier, voire à les monétariser, tout en sachant qu'aucune nuisance n'est jamais attribuable qu'aux transports (voir par exemple Héran, 2011a).

Plus délicate et presque jamais envisagée : la question des interrelations entre nuisances. Conformément à l'approche sectorielle, il est d'usage d'étudier chaque nuisance indépendamment des autres. Dans leurs recommandations, les économistes insistent sur la nécessité d'éviter «les doubles comptes» (Boiteux, 2001). Mais il convient tout autant d'explorer les effets de synergie entre les nuisances (certes, $2+2$ peut faire 3, mais aussi 5). Or l'ensemble des nuisances directes liées au transport converge vers quatre impacts indirects majeurs : la désaffection pour les modes non motorisés (et la dépendance aux modes motorisés), la réduction des relations de voisinage (et l'augmentation des relations lointaines), la dégradation de la santé et la dégradation du cadre de vie. Ces quatre impacts sont eux-mêmes pris dans des spirales négatives qui les renforcent (figure 1).

1/ La désaffection pour les modes actifs est d'abord due à l'insécurité routière et aux effets de coupure générés par les infrastructures de transport rapide. Elle est aussi liée à l'envahissement des espaces publics par les véhicules individuels motorisés, au bruit et à la pollution générés par le trafic et aux paysages dégradés par les véhicules et les infrastructures. Il en résulte un nécessaire accompagnement des personnes vulnérables et plus largement un report vers les modes motorisés, ce qui explique en partie la dépendance automobile (Dupuy, 1999).
2/ La diminution des relations de voisinage contribue à détériorer le lien social, à accroître le sentiment d'exclusion, surtout chez les bas revenus, et à compliquer l'accès aux services de proximité. Elle est en partie la conséquence des atteintes aux personnes et à leur environnement provoquées par les transports. Une étude célèbre, réalisée à San Francisco au début des années 1970, a bien montré que dans les rues les plus circulées, les relations de voisinage sont fortement réduites (Appleyard et al., 1981).

3/ Concernant la dégradation de la santé humaine, toutes les nuisances y concourent : l'insécurité routière, mais aussi la pollution à travers diverses maladies chroniques, le bruit source d'anxiété et de perturbation du sommeil, la dégradation des paysages et la restriction des espaces de vie sources de stress. De plus, la réduction des déplacements à pied et à vélo favorise les nombreuses maladies liées à la sédentarité. Et l'ensemble de ces impacts n'est pas sans conséquence sur la productivité du travail (voir les travaux de l'Onaps, Observatoire national de l'activité physique et de la sédentarité).

4/ La dégradation du cadre de vie est elle aussi en bonne partie la conséquence des multiples nuisances du transport : bruit, pollution, accidents, espaces réduits, déplacements de proximité limités, paysages abîmés. Dans les années 1960-1970, ce phénomène a favorisé l'exode des familles en périphérie. La modération de la circulation dans les centres-villes a d'ailleurs permis un retour des habitants.

Parmi les modes de déplacement, il se trouve que l'automobile est de loin celui qui génère le plus
Figure 1 : Le système de nuisances des transports en milieu urbain (Source : Héran, 2011b) system of nuisances

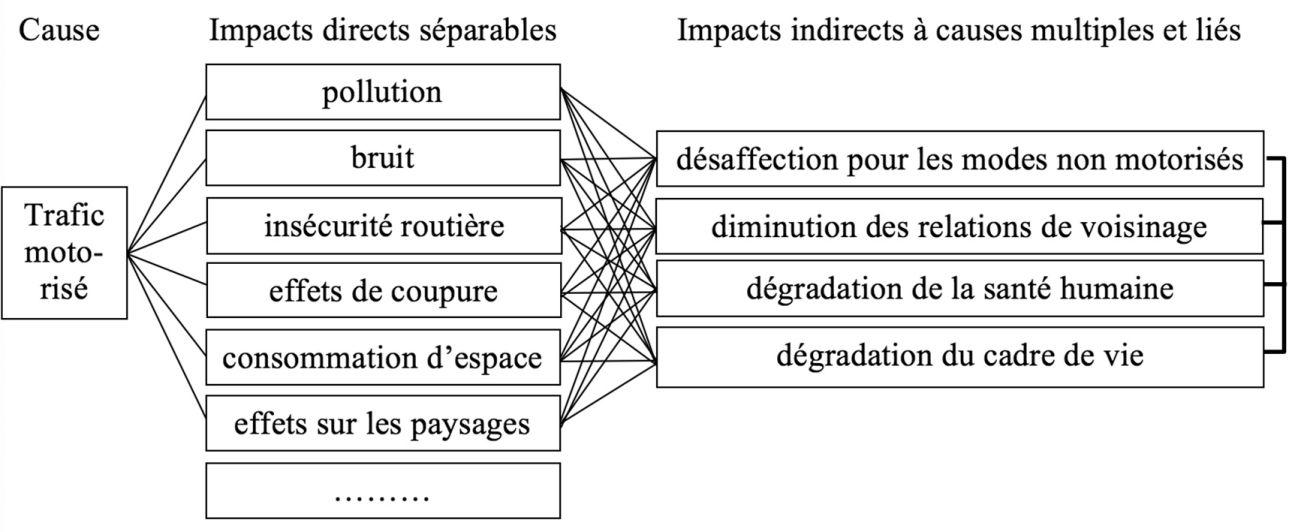

Source : Héran, 2011b. 
d'externalités négatives par personne transportée. Ce n'est pas un jugement de valeur mais un simple constat. L'objectif n'est pas de « stigmatiser» les automobilistes ou de les «culpabiliser » en leur tenant un discours «moralisateur » (Flonneau et Orfeuil, p. 14 et 137), mais de rendre compte d'une réalité de façon à la fois précise et pédagogique. C'est d'ailleurs une responsabilité éminente de la recherche académique, des organismes publics tels que l'ADEME ou le CEREMA et de certaines associations.

\section{LES IMPLICATIONS D'UNE APPROCHE OMNIMODALE}

De nombreuses conséquences découlent d'une approche plus globale des déplacements urbains, qu'il s'agisse de la nécessaire hiérarchisation des modes ou du rôle joué par les politiques de modération de la circulation automobile dans l'équilibre entre les modes. Seul un raisonnement en termes de paradigme permet de comprendre la portée de ces évolutions.

\section{Une hiérarchisation nécessaire des modes de déplacement}

Si les modes de déplacement ne concourent pas dans la même catégorie, tant leurs caractéristiques diffèrent, si certains modes génèrent beaucoup plus d'externalités négatives que d'autres, on ne peut laisser jouer la libre concurrence, car ce sont alors forcément les plus forts qui gagnent. Dans l'écosystème des déplacements urbains, l'espèce envahissante qu'est la voiture tend à étouffer les autres modes. C'est pourquoi, il convient de hiérarchiser les modes de déplacement selon leur degré de vulnérabilité et d'urbanité, en privilégiant, non plus l'automobile ou les transports publics, mais d'abord les modes actifs, puis les transports publics et enfin les modes individuels motorisés (Lévy, 2004). C'est à cette conclusion logique et très générale que parvient peu à peu un nombre croissant de villes.

Les piétons et les cyclistes sont en effet au fondement de l'urbanité. Ce sont eux qui animent la ville et la rendent plus sûre (Jacobs, 1961). La marche, et dans une moindre mesure le vélo, ne provoquent pas de nuisance, ni d'accident, ou très peu. Ces modes actifs contribuent en outre à améliorer la santé publique (Inserm, 2008). En n'ayant pas recours à des technologies sophistiquées, ils rendent les gens plus autonomes. Ils favorisent un usage égalitaire, libre et anonyme de la ville et de ses espaces publics. Ils sont, en ce sens, au fondement même de la démocratie (Paquot, 2009; Gehl, 2013).

Cependant, le but n'est pas de remplacer le tout automobile ou le tout transports publics par un «tout modes actifs » qui correspondrait à une priorité accordée à la marche et au vélo en toutes circonstances, car il va de soi qu'il est impossible de réaliser ainsi tous les déplacements, même avec l'essor actuel des VAE qui tend à doubler les distances parcourables à bicyclette. L'objectif est de trouver un équilibre entre l'ensemble des modes, de façon à préserver une liberté suffisante (et non pas totale) de se déplacer avec tous les modes de déplacement (et non pas seulement avec l'automobile).

\section{L'essor des politiques de modération de la circulation automobile}

Sur le plan historique, le tout automobile a concerné tous les pays, mais à des moments divers du fait du niveau de richesse atteint, mais aussi de l'existence ou non d'une industrie automobile nationale. Ainsi, les États-Unis ont ouvert la voie dès l'entre-deux-guerres avec leur production en chaîne de véhicules à bas coût. En revanche, les Pays-Bas n'ont développé une production locale de petites voitures avec Daf qu'à partir des années 1960; l'usage de la bicyclette s'est alors effondré comme partout ailleurs et au même rythme (Welleman, 1999).

Puis le tout automobile a commencé à être remis en cause dans les pays motorisés de longue date ou à la culture urbaine très ancienne. Dès les années 1960, les États-Unis connaissent une forte opposition à l'extension des autoroutes urbaines : the Freeway Revolt (Mohl, 2004), et depuis lors, plusieurs autoroutes vétustes ont été détruites et remplacées par des boulevards urbains. Le Congress for the New Urbanism en tient la chronique sur son site Internet. À la fin des années 1960, les PaysBas et l'Italie sont à leur tour touchés par des mouvements contestataires (Vàhl et Giskes, 1988). Les populations exigent un encadrement du trafic automobile pour éviter que les enfants qui jouent dans la rue ne se fassent écraser. Les Néerlandais inventent alors les cours urbaines - la première à 
Delft en 1969 - puis les zones 30 (Loiseau, 1975). Les Italiens instaurent des «zones à trafic limité » dans le centre de leurs villes - la première à Ferrare dès 1969 - (Lieutier, 1997). Depuis les années 1980, l'Allemagne généralise les zones 30, en élaborant le concept de modération de la circulation (Verkehrsberuhigung) à l'échelle de toute une agglomération (Umweltbundesamt, 1985).

La France n'est pas en reste, mais elle a suivi une voie originale. Ayant longtemps délaissé ses transports publics, elle s'est efforcée de rattraper son retard à partir des années 1970. Dans un premier temps, les métros de Lyon, Marseille et Lille, comme la première ligne du tramway de Nantes qui a repris le tracé d'une ancienne voie ferrée n'avaient pas pour but de remettre en cause le tout automobile. Mais dans des villes comme Grenoble et surtout Strasbourg, qui n'avaient pas les moyens d'investir dans un métro, il a fallu faire place au tramway en réduisant fortement le trafic automobile, puis reconquérir les espaces publics alentour. Cette politique a profité non seulement au transport public, mais aussi aux piétons et plus encore aux cyclistes.

À travers le jeu complexe d'un report modal général, la modération de la circulation automobile bénéficie en effet à tout le monde. Les transports publics profitent directement de la baisse du trafic et indirectement de l'essor de la bicyclette grâce au rabattement à vélo plutôt qu'à pied sur les stations terminus et à la désaturation des tronçons centraux des lignes. Les piétons sont plus en sécurité et les cyclistes accroissent la portée de leurs déplacements. Les usagers qui ont vraiment besoin d'une voiture se déplacent certes un peu moins vite, mais la vitesse moyenne est beaucoup moins réduite que la vitesse limite (de l'ordre de $5 \%$ à l'heure de pointe). La baisse de la vitesse, la limitation du stationnement de surface et l'essor des modes économes en espace autorisent la reconquête de nombreux espaces publics : les trottoirs peuvent être élargis, les carrefours redeviennent des places, les plantations se multiplient...

En prenant un recul suffisant, la tendance observable dans tous les pays développés est bien à la modération de la circulation automobile (vitesse et volume) et à l'essor des mesures en faveur des modes alternatifs à l'automobile (Héran, 2014). Elle concerne d'abord les grandes villes et les centres- villes, puis progressivement les proches périphéries et le centre des villes moyennes et enfin la grande périphérie. Si bien qu'il existe inévitablement d'importants décalages dans les situations observées à un moment donné, des accélérations comme des lenteurs dans les évolutions, et une diversité de trajectoires pour y parvenir.

Divers facteurs contribuent en effet à ralentir ou à accélérer le mouvement. Pour les populations qui ont accédé difficilement à la voiture individuelle, explorer d'autres façons de se déplacer paraît impensable. En revanche, les nouvelles générations manifestent un moindre attachement à la voiture. La mobilité résidentielle, qui facilite grandement le report modal, est environ deux fois moins forte en périphérie qu'au centre des grandes villes. La requalification des espaces publics et les aménagements en faveur des modes alternatifs à l'automobile ne sont guère à la portée des collectivités les plus pauvres. La reconstitution progressive d'un système vélo - comme il existe déjà un système automobile - devrait contribuer à rendre ce mode plus attractif. Une relocalisation de la distribution plus proche des clients peut aussi relancer les achats à pied ou à vélo. Les plans de déplacement d'entreprise désormais obligatoires pour les entreprises de plus de 100 salariés peuvent stimuler les nouvelles mobilités. Enfin, il existe parfois des reculs suite à des changements de majorité municipale, mais qui ne sont le plus souvent que conjoncturels.

Tout est finalement une question de rythme du changement et de recul suffisant. Ainsi, la part modale du vélo augmente, dans toutes les villes calmées des pays développés, au rythme très rapide d'environ $10 \%$ par an (soit un doublement tous les 7 ans), comme c'est le cas par exemple dans les grandes villes américaines (NYC DoT, 2016). Elle est passée à Paris de seulement 0,2 \% en 1976 à $5 \%$ aujourd'hui (source: Mairie de Paris) et elle devrait dépasser la part modale de la voiture avant 2030. De même, la part modale des 2RM commence souvent par croître avec la restriction du trafic automobile, mais finit toujours par décroître à cause du risque considérable d'accidents avec un tel mode, qui décourage nombre d'usagers. Le VAE constitue depuis peu une alternative beaucoup plus sûre. Ainsi, Paris a connu un doublement des déplacements en 2RM entre 1991 et 2007, puis une stabilisation depuis 2008, dans un contexte d'effon- 
drement des ventes de 2RM en France (- $44 \%$ entre 2007 et 2015).

\section{Un changement de paradigme}

Pour comprendre ces évolutions profondes et les divers décalages repérés, il convient de raisonner en termes de changement de paradigme (Kuhn, 1962) et non de "modèle urbain » (Carriou et Ratouis, 2014) ou de "schéma de mobilité » (Bieber et Orfeuil, 1993).

Le changement de paradigme se joue simultanément à plusieurs niveaux. Sur le plan de la mobilité, au tout automobile tend à succéder partout une « voirie pour tous » (CNT, 2005) ou des « rues pour tous » préfèrent dire les Allemands (Monheim, 1990) ou les Anglo-saxons (European Commission, 2004). En termes de méthode, l'approche sectorielle, monomodale laisse peu à peu place à une approche systémique, omnimodale qui conçoit d'emblée l'articulation entre tous les modes. Enfin, à l'urbanisme moderne, qui sépare les fonctions et les circulations, se substitue progressivement une ville plus durable, cherchant à limiter l'étalement urbain et à mélanger les fonctions urbaines pour permettre à l'ensemble des modes de déplacement de cohabiter à petite vitesse sur des distances plus courtes.

Ce cadre d'analyse transcende les distinctions que proposent certains auteurs entre "modèle saintsimonien », « modèle californien » et « modèle rhénan » (Bieber et al., 1993), entre «ville consolidée », «ville intermédiaire » et «ville territoire » (Masboungi, 2015). Certes, les dimensions sociologiques, culturelles et institutionnelles propres à chaque pays, à chaque agglomération ou à chaque commune ont une influence incontestable sur l'organisation des déplacements. Mais le changement de paradigme explique déjà une bonne part des évolutions passées et probables du système des déplacements urbains.

Il est erroné de considérer que les territoires périurbains sont à jamais colonisés par le tout automobile. Même en France, les politiques de «voirie pour tous » s'étendent en périphérie des grandes villes et dans les villes moyennes. Certains bourgs requalifient leur centre et généralisent les zones 30 aux quartiers pavillonnaires. Des périurbains réclament l'existence de trottoirs le long des rues, protégés du stationnement illicite, ainsi qu'une modération générale des vitesses, pour que leurs enfants puissent se rendre seuls à l'école ou à leurs activités à pied. On peut montrer que des territoires peu denses offrent un potentiel non négligeable aux mobilités alternatives (Brès, 2015).

Certes, le raisonnement en termes de changement de paradigme paraît assez déterministe. Pourtant, personne ne peut nier que la voiture individuelle a séduit la planète entière. La facilité de déplacement qu'elle offre est irrésistible, malgré les externalités négatives générées. La prise de conscience des dégâts que son libre développement provoque n'en est pas moins universelle. Chaque pays et chaque ville veulent eux-mêmes s'en rendre compte. La nécessité d'y remédier et de redonner toute leur place aux autres modes émerge ensuite partout. Mais pour parcourir toutes ces étapes, les trajectoires sont infiniment diverses et elles doivent s'accorder avec d'autres évolutions : en matière d'urbanisme, d'approches méthodologiques, de mentalités... Le déterminisme n'est donc pas linéaire mais systémique (Brun, 2006, p. 146-157).

Un changement de paradigme provoque toujours de vives tensions. Les constructeurs automobiles, les entreprises de travaux publics, les bureaux d'études et les institutions qui ont construit leurs compétences sur l'ancien paradigme cherchent des solutions pour prolonger son existence le plus longtemps possible, afin de sauvegarder leurs intérêts. Les voitures hybrides, électriques ou autonomes, la route intelligente, de nouveaux barreaux autoroutiers peuvent être considérées comme de telles tentatives. Pourtant, en continuant à consommer des ressources de plus en plus rares sans réduire vraiment les nuisances, elles constituent autant des problèmes que des solutions. Les rares acteurs qui choisissent de travailler sur le nouveau paradigme apparaissent assez décalés et sont d'abord ignorés, puis fermement contrés. Le dialogue est difficile car chacun vit dans un autre monde. Kuhn parle d'incommensurabilité entre les paradigmes.

\section{Conclusion}

Dans les années de croissance d'après-guerre, le tout automobile a été une réponse logique à une aspiration légitime de la population à plus de mobilité et à un certain desserrement urbain. Mais les conséquences néfastes d'un usage excessif de l'auto- 
mobile n'ont pas été correctement estimées et il faut le plus souvent que les populations les découvrent elles-mêmes et s'en alarment pour que les autorités réagissent. Cette prise de conscience finit toujours par émerger et des mesures par être prises et cela dans tous les contextes. Pour s'en convaincre, il faut accepter cependant de prendre un recul historique suffisant et de s'intéresser à tous les pays développés. Les changements passés et à venir ne sont perceptibles que sur longue période et grâce à des comparaisons internationales.

Pour mesurer toute l'ampleur de ces changements, il est indispensable d'adopter une approche à la fois systémique, c'est-à-dire capable d'aborder tout le système des déplacements urbains, et omnimodale, c'est-à-dire capable de prendre en compte tous les modes de déplacement. Il devient alors possible de révéler l'inégalité des modes dans la concurrence qu'ils se livrent entre eux et les énormes écarts entre les externalités négatives qu'ils génèrent. Dès lors, peut-on esquisser une représentation de l'avenir - une vision - d'une tout autre ambition que celle visant à infléchir les tendances passées, tout en cherchant à satisfaire tout le monde, non sans de nombreuses contradictions.

Pour élaborer une politique de déplacements cohérente, le premier principe est de protéger d'abord les plus faibles et les plus urbains, soit les piétons et les cyclistes. Ce qui impose une modération des vitesses puis du trafic lui-même qui débouche sur une hiérarchisation moindre et un meilleur maillage des réseaux. Le deuxième principe est de réduire les externalités négatives des transports de façon à sortir des nombreux cercles vicieux dans lesquels elles enferment les acteurs : ne plus dépendre exclusivement de l'automobile ou des transports publics, favoriser les relations de voisinage, améliorer la santé publique et le cadre de vie. Le troisième principe est d'offrir une liberté de déplacement au plus grand nombre et non pas seulement aux automobilistes qui ont souvent confisqué de fait cette liberté à leur seul profit. Le quatrième principe pourrait être la reconquête des espaces publics pour redonner de l'urbanité à la ville, y compris en périphérie, en réduisant notamment au maximum le stationnement automobile sur voirie. Tous ces principes - et on pourrait en ajouter d'autres - sont étroitement liés entre eux et se renforcent les uns les autres. C'est précisément ce qui constitue un nouveau paradigme.
Quant à la mise en œuvre de cette vision, c'est d'abord une question de rythme et de concertation. Il n'est pas question de tout changer du jour au lendemain. Les populations, les commerçants, les employeurs doivent être associés au diagnostic, puis au choix des solutions et disposer de temps pour s'adapter. Si les principes sont d'emblée bien posés, remis dans une perspective historique et planétaire, les politiques de déplacement qui en découlent sont ensuite bien plus cohérentes, tout en s'adaptant à l'infinie variété des contextes.

\section{Bibliographie}

Appleyard D., Gerson M. S., Lintell M., 1981. Livable Streets, Berkeley, University of California Press, 364 p.

Bieber A., Massot M.-H., Orfeuil J.-P., 1993. Questions vives pour une mobilité quotidienne, rapport pour la DATAR, synthèse INRETS, 19, 76 p.

Bieber A., Orfeuil J.-P., 1993. La mobilité urbaine et sa régulation. Les annales de la recherche urbaine, 59-60, p. 127139.

Bigey M., Schmider A., 1971. Les transports urbains, Paris, Éd. universitaires, $186 \mathrm{p}$.

Boiteux M. (dir.), 2001. Transports : choix des investissements et coût des nuisances, rapport pour le Commissariat général du Plan, Paris, La Documentation française, 325 p.

Brès A., 2015. Figures discrètes de l'urbain, à la rencontre des réseaux et des territoires, Genève, Métis Presses, 172 p.

Brun G., 2006. Introduction à l'histoire totale, Paris, Economica coll. «Anthropos », 465 p.

Buhler T., 2015. Déplacements urbains : sortir de l'orthodoxie. Plaidoyer pour une prise en compte des habitudes, Lausanne, Presses polytechniques et universitaires romandes, $123 \mathrm{p}$.

Carriou C., Ratouis O., 2014. Quels modèles pour l'urbanisme durable?, Métropolitiques, 25 juin, 7 p.

Cerema, 2015. Évaluation a posteriori des transports collectifs en site propre. Note méthodologique, Lyon, Cerema, 104 p.

Certu, 2002. Evaluation des transports en commun en site propre. Recommandations pour l'évaluation socio-économique des projets de TCSP, Lyon, Certu, $144 \mathrm{p}$.

Certu, 2012. La mobilité urbaine en France : enseignement 2000-2010, Lyon, Certu, 108 p.

Conseil national des transports, 2005. Une voirie pour tous. Sécurité et cohabitation sur la voie publique au-delà des conflits d'usage, Paris, CNT, 2 tomes.

De Lapparent M., 2013. Les modèles occupation du sol-transport comme outils d'évaluation, contribution au rapport du groupe de travail présidé par É. Quinet sur L'évaluation socio-économique en période de transition, Commissariat général à la stratégie et à la prospective, 29 p. 
Devillers C., 1994. Le projet urbain, in Conférences Paris d'architectes 1994, Paris, Éditions du Pavillon de l'Arsenal, p. 7-47.

Dupuy G., 1975. Une technique de planification au service de l'automobile: les modèles de trafic urbain, Paris, ATP, 201 p.

Dupuy G., 1999. La dépendance automobile. Symptômes, analyses, diagnostic, traitements, Paris, Anthropos, 160 p.

European Commission, 2004. Reclaiming city streets for people. Chaos or quality of life?, Luxembourg, Office for Official Publications of the European Communities, $52 \mathrm{p}$.

FAIVRE D'Arcier B., 2010. La situation financière des transports publics urbains est-elle « durable »?, Les Cahiers scientifiques $d u$ transport, 58, p. 3-28.

Flonneau M., Orfeuil J.-P., 2016. Vive la route, vive la République!, La Tour-d'Aigues, Éditions de L’Aube, 162 p.

Gent J., 2013. Pour des villes à échelle humaine, Montréal, Éditions Écosociété, 273 p.

Gerondeau C., 1977. Les transports urbains, Paris, PUF, coll. "Que sais-je? », $2^{\mathrm{e}}$ éd., 127 p.

Goodwin P.B., 1998. Unintended effects of transport policies, in BAnister D. (ed.), 1998, Transport policy and the environment, London, E \& FN Spon, p. 114-130.

Goodwin P. B., 2003. Unintended effects of policies, in Hensher D. A., Button K. J. (ed.), Handbook of Transport and the Environment, Elsevier Ltd., chapter 7, p. 603-613.

HÉrAn F., 201 la. La ville morcelée. Effets de coupure en milieu urbain, Paris, Economica, 218 p.

Héran F., 201 lb. Pour une approche systémique des nuisances liées aux transports en milieu urbain, Les Cahiers scientifiques du transport, 59, p. 83-112.

HÉrAn F., 2013. La consommation d'espace-temps des transports en milieu urbain, in Brun G. (éd.), Ville et mobilité. Nouveaux regards, Paris, Economica, coll. « Méthodes et approches », p. 177-191.

HÉRAN F., 2014. Le retour de la bicyclette. Une histoire des déplacements urbains en Europe de 1817 à 2050, La Découverte, Paris, coll. «Cahiers libres », 256 p.

Inserm, 2008. Activité physique. Contextes et effets sur la santé, Paris, Éditions Inserm, 832 p.

Jacobs J., 1961. The Death and Life of great American Cities, trad. Déclin et survie des grandes villes américaines, Liège, Pierre Mardaga, 1991, 435 p.

Kunn T. S., 1962. The structure of Scientific Revolutions, trad. La structure des révolutions scientifiques, Paris, Flammarion, $285 \mathrm{p}$.

Le Moigne J.-L., 1977. La théorie du système général, théorie de la modélisation, Paris, PUF, 258 p.

LÉvy J., 2004. Modèle de mobilité, modèle d'urbanité, in Allemand S., Ascher F., Lévy J. (dir.), Les sens du mouvement, Paris, Belin, p. 157-169.

Lieutier G., 1997. Déplacements urbains. Les solutions mises en œuvre dans les villes italiennes : les ZTL, zones à trafic limité, Transports urbains, 94, p. 5-14.
Loiseau F., 1975. Intégration de trafic et habitat urbain. L'expérience de Delft, Transport environnement circulation, 12, p. 24-30.

Masboungi A. (dir.), 2015. Ville et voiture, Marseille, Parenthèses, coll. «Projet urbain », 191 p.

Mont R. A., 2004. Stop the Road, Freeway Revolts in American Cities, Journal of Urban History, 30 (5), p. 674-706.

Monheim H., Monheim-Dandorfer R., 1990, Straßen für alle. Analysen und Konzepte zum Stadtverkehr der Zukunft, Hamburg, Rasch und Röhring, 530 p.

New York City Department of Transportation, 2016. Cycling in the City. Cycling Trends in NYC, New York, NYC-DoT, $20 \mathrm{p}$.

Offaner J.-M., 2006. Les plans de déplacements urbains, Paris, La Documentation française, $96 \mathrm{p}$.

Paquot T., 2009, L'espace public, Paris, La Découverte, coll. « Repères », 125 p.

Paul-Dubois-Taine O. (dir.), 2010. Les nouvelles mobilités: adapter l'automobile aux modes de vie, rapport pour le Centre d'analyse stratégique, $135 \mathrm{p}$.

Paul-Dubois-Taine O. (dir.), 2012. Les nouvelles mobilités dans les territoires périurbains et ruraux, rapport pour le Centre d'analyse stratégique, $130 \mathrm{p}$.

Poulit J., 1971. La circulation urbaine, Revue générale des routes et des aérodromes, 462, p. 59-78.

Reigner H., Brenac T., Hernandez F., 2013. Nouvelles idéologies urbaines. Dictionnaire critique de la ville mobile, verte et sûre, Rennes, Presses universitaires de Rennes, 178 p.

SmeEd R. J., 1961. The traffic problem in towns, traduction Le problème de la circulation dans les villes, Arcueil, SERC, $1965,59 \mathrm{p}$.

Umweltbundesamt, Kolloquium Forschungsvorhaben Flächenhafte Verkehrsberuhigung [(Bureau fédéral de l'environnement, Colloque sur les projets de modération de la circulation à grande échelle], Berlin, 1985.

VÀHL H. G., GisKes J., 1988. Urbanisme et trafic, de la guerre à la paix, Bagneux, CETUR, 48 p.

Welleman T., 1999. The Dutch bicycle masterplan. Description and evaluation in an historical context, The Hague, The Ministry of Transport, Public Works and Water Management, $130 \mathrm{p}$.

WiEl M., 1999. La transition urbaine ou le passage de la ville pédestre à la ville motorisée, Liège, Pierre Mardaga, 149 p.

Wiel M., 2007. Pour planifier les villes autrement, Paris, L'Harmattan, $244 \mathrm{p}$.

Wiel M., 2015. Grand Paris. Vers un plan B, Paris, La Découverte, $420 \mathrm{p}$.

Zahavi J., 1973. The TT-relationship: a unified approach to transportation planning, Traffic Engineering and Control, 15 (4-5), p. 205-212. 